\title{
Variación del canto de Myiothlypis fulvicauda (Parulidae) en ambientes naturales de Ibagué (Tolima, Colombia)
}

\author{
Song Variation of Myiothlypis fulvicauda (Parulidae) in Natural \\ Environments in the municipality of Ibagué (Tolima, Colombia)
}

\section{Daniela Villamizar-Soto ${ }^{\text {ab }}$, Edwin Orlando López-Delgado ${ }^{\text {ac }}$, Sergio Losada-Prado ${ }^{\text {ad }}$}

\footnotetext{
a Grupo de Investigación en Zoología (GIZ), Programa de Biología, Facultad de Ciencias, Universidad del Tolima, Colombia.

bdvillamizars@ut.edu.co | https://orcid.org/0000-0002-6272-638X

chttps://orcid.org/0000-0002-4010-1880

${ }^{\mathrm{d}}$ https://orcid.org/0000-0001-6916-3893
}

\section{RESUMEN}

El canto de las aves es fundamental para la comunicación y el desarrollo de diferentes actividades. Por otra parte, el ruido que proviene de diversas fuentes dificulta su recepción, por lo cual algunas aves cambian la estructura, frecuencia, energía o el horario de su canto con el fin de asegurar ser escuchadas. Particularmente, el ruido generado por ríos es constante y tiene mayor intensidad a bajas frecuencias, por lo que puede enmascarar el canto. Por consiguiente, el objetivo de este trabajo fue analizar el canto de Myiothlypis fulvicauda en ambientes con ruido natural generado por cuerpos de agua en el municipio de Ibagué (Tolima), teniendo en cuenta además la temporada climática y el grado de cobertura vegetal. Las grabaciones se realizaron en los meses de mayo, julio y octubre de 2019 en 8 localidades, estableciendo dos tratamientos de ruido: alto y bajo. Se emplearon modelos lineales generalizados, así como análisis de varianza y correlación, para evaluar el efecto del ruido, la temporada climática y el grado de cobertura vegetal sobre el canto de esta ave. Se encontró que la frecuencia máxima, el rango de frecuencias y el número de silbidos iniciales fueron mayores en hábitats con ruido alto. A su vez,

Citation: Villamizar-Soto, D., López-Delgado, E. O. y Losada-Prado, S. (2021). Variación del canto de Myiothlypis fulvicauda (Parulidae) en ambientes naturales de Ibagué (Tolima, Colombia). Mutis, 11(2), 22-32. https://doi.org/10.21789/22561498.1733

Recibido: 6 de abril de $2021 \quad$ Aceptado: 15 de mayo de 2021

Copyright: $\odot 2021$. Villamizar-Soto, D., López-Delgado, E. O. y LosadaPrado, S. (2021). This is an open-access article, which permits unrestricted use, distributions and reproduction in any medium, provided the original author and source are credited.

Competing Interests: The authors have no conflict of interest. el rango de frecuencias fue menor durante la temporada seca. Por su parte, el número de elementos repetitivos y las frecuencias máximas también fueron menores en localidades con vegetación más densa. Estos cambios en el canto de $M$. fulvicauda denotan una posible adaptación de la especie como respuesta al ruido y a características de su hábitat tales como la cobertura vegetal y la temporada climática.

Palabras clave: bioacústica, adaptación acústica, vocalización, arañero ribereño, ruido. 


\section{ABSTRACT}

Bird songs are fundamental for the communication and development of different bird activities. On the other hand, the noise coming from various sources make it difficult to receive bird songs, and therefore some bird species have changed the structure, frequency, energy, or time of their singing to ensure they can be heard. Particularly, the noise generated by rivers is constant and has greater intensity at low frequencies, so it could mask birds' songs. Hence, the objective of this work was to examine the song of Myiothlypis fulvicauda in environments with natural noise generated by water bodies in the municipality of Ibagué (Tolima), considering the climatic season and the degree of vegetation cover within this analysis. Recordings were performed during May, July, and October 2019 at 8 locations, which were assigned two types of noise treatment: high and low. Generalized linear models and variance and correlation analysis were used to evaluate the effect of noise, climatic season, and vegetation cover degree on the singing of this species of bird. We found that the maximum frequency, frequency bandwidth, and number of initial whistles were higher in high-noise habitats, while the frequency bandwidth was lower during the dry season. Besides, the number of repetitive elements and maximum frequencies were lower in highly dense vegetation locations. These changes in the song of $M$. fulvicauda denote a possible adaptation of this species in response to environmental noise and habitat characteristics such as vegetation cover and season patterns.

Keywords: Bioacoustics, acoustic adaptation, vocalization, Buff-rumped Warbler, noise.

\section{INTRODUCCIÓN}

Las aves usan cantos y llamados para comunicarse, por lo que estas vocalizaciones cumplen funciones importantes como avisar sobre alimento y peligro, la defensa del territorio y atraer pareja, entre otras (Catchpole \& Slater, 2008). Los cantos, por lo general, son largos, complejos y producidos por machos, aunque en el trópico es común que también los produzcan las hembras. Los llamados, en cambio, suelen ser cortos, simples y producidos por ambos sexos (Gill, 2007). Por ello, la correcta emisión y recepción del canto permite al macho tener mayor éxito reproductivo y defensa de su territorio. A su vez, las hembras podrán elegir al macho cuyo canto demuestre que este será capaz de brindar a su descendencia las mejores características para el lugar que habitan (Catchpole \& Slater, 2008).

En la naturaleza existe una gran variedad de fuentes de ruido, entre las que se cuentan las cascadas y los ríos (abióticas), cuya energía sonora se concentra en las frecuencias más bajas y puede enmascarar el canto de las aves (Narins et al., 2004). Otras fuentes de ruido que enmascaran el canto corresponden a fuentes bióticas como el canto de otras aves, debido a la similitud espectral y temporal de los sonidos entre algunas especies (Brumm, 2013). Así mismo, con la continua expansión urbana, los sonidos que produce el ser humano constituyen otra fuente de ruido (antropogénica) (Brumm, 2013; Pijanowski et al., 2011). Por otra parte, cuando el canto es emitido este se propaga a través de un medio, por lo que factores como el aire, el suelo y la vegetación pueden reducir su energía mediante absorción, o redirigirlo gracias a la reflexión o difracción tras chocar con algún elemento, de manera que estos fenómenos acústicos también pueden distorsionar el canto de las aves (Morton, 1975).

Los estudios existentes se han enfocado principalmente en evaluar el efecto del ruido antropogénico sobre el canto de las aves, encontrando pocas investigaciones que incluyan fuentes de ruido naturales, aun cuando, por ejemplo, los ríos generan ruido constante que podría ser una fuerza selectiva que module la evolución de señales acústicas como el canto (Vargas-Salinas et al., 2014). Ciertos estudios han demostrado que en ambientes con altos niveles de ruido algunas aves no logran identificar a los invasores ni a su pareja (Swaddle $\&$ Page, 2007). Sin embargo, otras especies consiguen ser escuchadas a pesar del ruido gracias a la incorporación de cambios en la estructura de su canto, como incrementos en su frecuencia (Brumm \& Slabbekoorn, 2005; Douglas \& Conner, 1999; Slabbekoorn \& Peet, 2003), intensidad (Brumm, 2004), complejidad o duración (Brumm \& Slater, 2006). No obstante, estos cambios pueden afectar a los individuos, puesto que, en ocasiones, aunque el canto resulta más atractivo para las hembras, este puede ser menos efectivo para defender y mantener el territorio (Fernández-Juricic, 2005; Luther et al., 2016). 
Este trabajo tomó como objeto de estudio a la especie Myiothlypis fulvicauda (Parulidae), conocida como arañero ribereño, la cual se caracteriza por no presentar dimorfismo sexual, tener una coloración oliva pardusca, una rabadilla amarillenta brillante y en su parte inferior presentar una coloración blanca anteada a blanquecina (Hilty \& Brown, 2001). La especie está asociada a cuerpos de agua y suele verse en grupos de dos a tres individuos, en los que solo uno (aparentemente el macho) canta activamente durante todo el año (Escalante, 2013; Sánchez-Guzmán \& Losada-Prado, 2016).

Teniendo en cuenta lo expuesto, el objetivo de esta investigación fue analizar los posibles cambios en el canto de Myiothlypis fulvicauda como respuesta a la temporada climática, la cobertura vegetal y el ruido producido por quebradas en el municipio de Ibagué (Tolima, Colombia). Se espera que en ambientes con alto nivel de ruido el canto presente una frecuencia más alta, lo que se considera debe ocurrir en temporada lluviosa, cuando el ruido aumenta por el incremento de los caudales. Así mismo, se espera que en ambientes con mayor cobertura vegetal se presente un menor número de notas en el canto.

\section{MATERIALES Y MÉTODOS}

\section{Área de estudio}

El municipio de Ibagué (Tolima) está ubicado en la vertiente oriental de la Cordillera Central de Colombia, en su confluencia con el valle del Río Magdalena. Allí se encuentran paisajes de montaña con altas pendientes y la planicie conocida como meseta de lbagué (SPT, 2014). Este municipio está localizado a $1.285 \mathrm{~m}$ de elevación y una temperatura promedio de 21 ㄷ (SPT, 2014).

En esta zona se muestrearon ocho localidades en los alrededores del casco urbano (figura 1), en las que se evidenció la presencia de ríos, quebradas, canales de riego y aguas con corriente imperceptible, los cuales generan ruido de diferente intensidad. Estas localidades comprendieron dos zonas de vida, bosque seco tropical (Bs-T) y bosque húmedo premontano (BhPM) (Holdridge, 1967), y se caracterizan por ser áreas con poca intervención humana, alta presencia arbustiva y relictos de bosque secundario. Las localidades estudiadas fueron agrupadas de acuerdo con dos niveles de ruido: alto ( $>65 \mathrm{~dB}$ ) y bajo $(<65 \mathrm{~dB})$.

Figura 1. Localidades estudiadas en el municipio de Ibagué (Tolima)

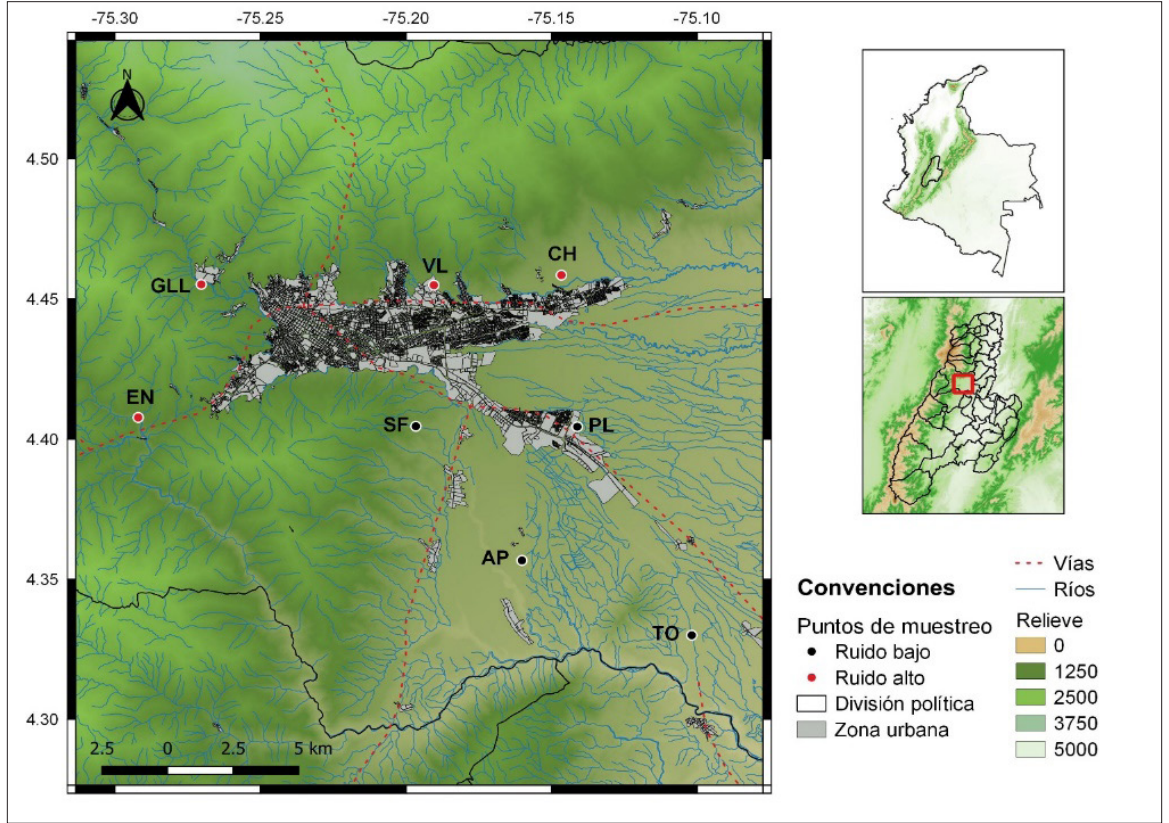

Río Coello (EN), Quebrada El Gallo (GLL), Quebrada Las Panelas (VL), Quebrada Chembe (CH), Quebrada Reserva Natural Santa Fe de los Guaduales (SF), Quebrada Aparco (AP), Canal de riego Hacienda el Escobal (PL) y Laguna el Toro (TO). 


\section{Recolección de datos}

Cada localidad fue visitada durante las temporadas lluviosa (mayo y octubre) y seca (julio) de 2019. Debido a que en el mes de mayo no fue posible obtener las grabaciones requeridas en todas las localidades, los puntos restantes fueron visitados en octubre. Las grabaciones fueron realizadas por un solo investigador entre las 06:00 y 11:00 horas, para lo cual se hicieron recorridos paralelos a los cuerpos de agua, donde se grabaron los cantos de $M$. fulvicauda, procurando tener a la vista del observador al individuo emisor. Una vez grabado un individuo, el desplazamiento se hizo en dirección opuesta a la que este siguió para evitar grabarlo nuevamente. Para esta actividad se utilizó una grabadora Marantz PMD661, un micrófono omnidireccional Sennheiser ME-62 y una antena parabólica Sennheiser, configurada a una tasa de muestreo de $44.100 \mathrm{~Hz}$ y codificación de 16 bits.

Para el ruido se tuvieron en cuenta las diferentes caídas de agua, tomando mediciones durante dos horas (06:00h - 08:00 h) para obtener el nivel de presión sonora continuo equivalente $\left(L_{\text {Aeq }}\right)$, empleando un sonómetro Extech 407730 con una ponderación de frecuencia A y una respuesta rápida (Londoño \& Fernández, 2011). Además, se obtuvo el grado de cobertura vegetal de cada localidad, el cual se refiere al espacio ocupado por las plantas (Melo \& Vargas, 2002). Para ello, se elaboraron tres parcelas de $10 \times 20$ $\mathrm{m}$ perpendiculares a los cuerpos de agua y se tuvieron en cuenta todas las plantas con diámetro a la altura del pecho igual o superior a $3 \mathrm{~cm}$ (Sánchez-Rodríguez et al., 2003).

\section{Análisis de datos}

Se realizaron cortes de las grabaciones y se generaron espectrogramas en el software Raven Pro 1.5, en los que se configuró un tamaño de transformación de 512 y un tipo de ventana Hann (CCB, 2014). En todas las localidades se grabaron dos individuos, excepto en $\mathrm{AP}$, donde se realizó la grabación de tres individuos. De cada individuo se tomó el primer y último canto de la grabación con mejor calidad. Se analizaron las dos partes del canto, es decir, los silbidos iniciales (SI) -con menor rango de frecuencias-y los elementos repetitivos (ER) - notas similares de corta duración (forman un trino) con mayor rango de frecuencias $y$ amplitud hacia el final del canto- (Escalante, 2013). De estas partes se midió el número de notas totales (N_notas), número de SI (N_SI) y la duración de cada uno (D_SI), duración total del canto (D_total), velocidad de ER (Rate), frecuencia mínima y máxima de cada nota y del canto (Fmin, F_max), rango de frecuencias ( $F \_$rango) y frecuencia central (F_central), obteniendo un total de 118 variables correspondientes a cada medición (figura 2). Las variables de frecuencia se obtuvieron en $\mathrm{Hertz}(\mathrm{Hz})$ y el tiempo en segundos $(\mathrm{s})$.

Figura 2. Especie objeto de estudio: A. Estructura del canto de Myiothlypis fulvicauda; B. Adulto de M. fulvicauda

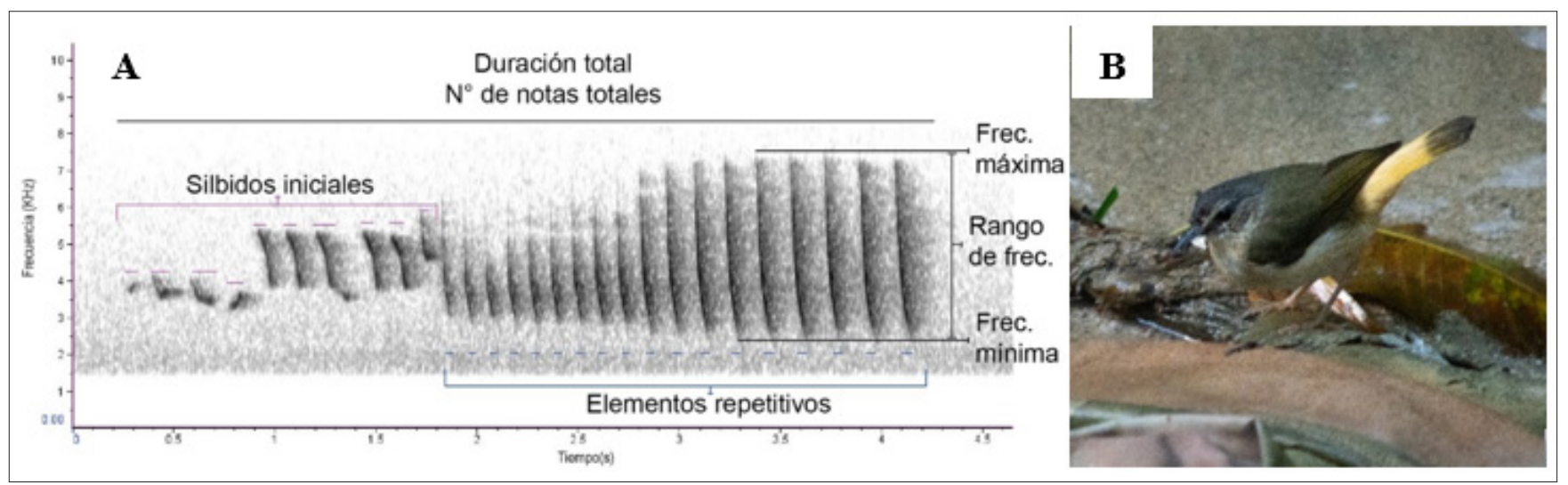

Fuente: elaboración propia. Fotografía: Daniela Villamizar-Soto. 
Para identificar diferencias en las variables del canto en los tratamientos de ruido y temporadas climáticas, se utilizó un análisis de varianza no paramétrico de Kruskal-Wallis, debido a que las variables no cumplieron con los supuestos paramétricos (normalidad y homogeneidad de varianza) esperados. El análisis de varianza se complementó con gráficos exploratorios de cajas y bigotes, en los cuales se representaron las variables generales del canto. Para identificar posibles relaciones entre la cobertura vegetal y las variables del canto, se empleó un análisis de correlación de Spearman.

Con el fin de realizar análisis multivariados, se evaluó la multicolinealidad de las variables del canto con un análisis de correlación de Spearman, eliminando aquellas variables con alta correlación (> $70 \%$ ). Posteriormente, se realizó un análisis de componentes principales (PCA) con el objetivo de seleccionar las variables de mayor contribución a ser utilizadas en los modelos lineales generalizados (GLM). Para los GLM se incluyeron como variables dependientes el rango de frecuencias del canto (F_rango), la frecuencia mínima del segundo ER (Fmin_2), la frecuencia central del canto ( $F$ _central), la frecuencia máxima del segundo silbido inicial (Fmax_SI2) y el número de silbidos iniciales (N_SI). Por su parte, el ruido, la temporada climática y la cobertura vegetal

Figura 3. Diagrama de cajas y bigotes de los tratamientos de ruido y las variables frecuencia mínima (F_min), frecuencia máxima (F_max), rango de frecuencias (F_rango), número de silbidos iniciales (N_SI), número de elementos repetitivos (N_ER) y frecuencia central (F_central)
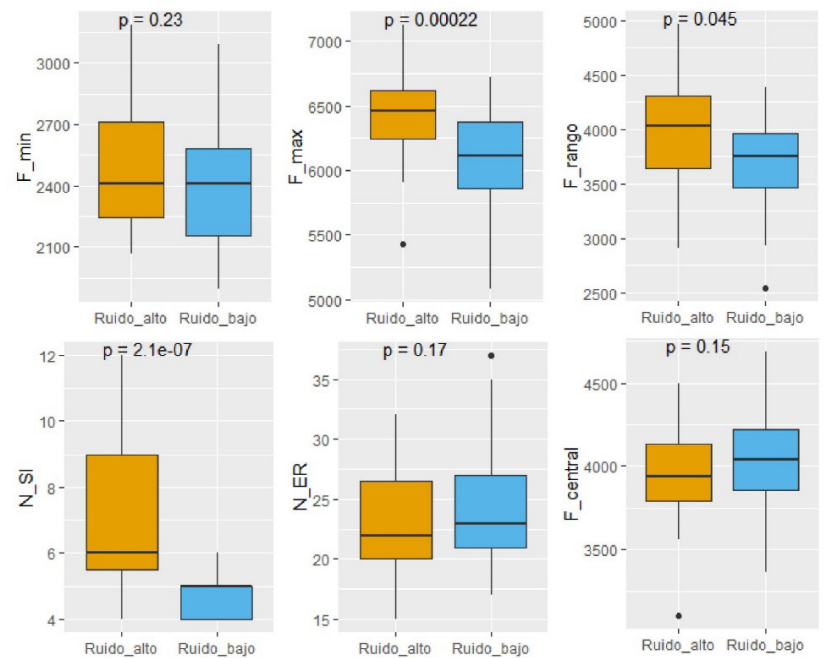

Fuente: elaboración propia. fueron establecidas como variables independientes, realizando diferentes combinaciones en las que se incluyeron una, dos o tres de estas variables en cada modelo. El mejor modelo fue escogido bajo el criterio de información de Akaike. Los análisis estadísticos se realizaron en el programa $R$ y las librerías Vegan y Ime4 (R Core Team, 2019).

\section{RESULTADOS}

Se analizaron 67 cantos, 33 de ellos en temporada seca y 34 en temporada lluviosa, 31 en localidades con ruido alto y 36 con ruido bajo. El número de notas registradas en estos osciló entre 22 y 41, con un promedio de $6 \mathrm{SI}, 24 \mathrm{ER}, 4,37 \mathrm{~s}$ de duración y una frecuencia del canto entre $1.894,9 \pm 296,3$ y $7.125 \pm$ $399 \mathrm{~Hz}$. Los análisis mostraron que 14 variables fueron diferentes entre los tratamientos de ruido y entre temporadas climáticas. En los tratamientos de ruido, las variables F_max, F_rango y N_SI fueron significativamente diferentes (figura 3), mientras que en las temporadas climáticas la F_min, F_rango y F_central fueron las variables que mostraron esta misma tendencia (figura 4). Con relación a la cobertura vegetal, se encontró una correlación inversa (40-60 \%) con las frecuencias máximas de los primeros siete $E R$, el número de ER y el número de notas totales.

Figura 4. Diagrama de cajas y bigotes de las temporadas climáticas y las variables frecuencia mínima (F_min), frecuencia máxima (F_max), rango de frecuencias ( $F_{-}$rango), número de silbidos iniciales (N_SI), número de elementos repetitivos (N_ER) y frecuencia central (F_central)
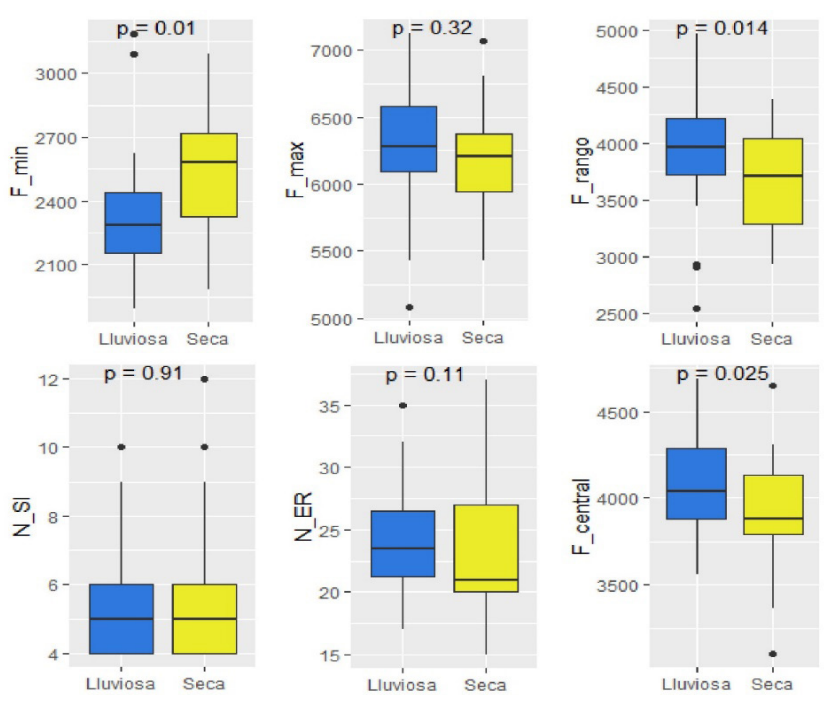

Fuente: elaboración propia. 
Las variables seleccionadas para los GLM se ajustaron a una distribución gamma, excepto en el caso del N_SI, la cual se ajustó a una distribución poisson. La tabla 1 resume los modelos seleccionados de las combinaciones realizadas para cada variable del canto. Con relación al F_rango, la temporada climática y el ruido influyeron de forma significativa; en localidades con ruido alto el F_rango fue mayor y en la temporada seca este disminuyó. Para la F_min, la cobertura vegetal mostró una relación inversamente proporcional. En cuanto a la F_central, de acuerdo con los resultados de los modelos durante la temporada seca, los valores de esta variable disminuyeron. En el caso de la Fmax_SI2, los valores de esta fueron mayores en las localidades con ruido alto y menores en las de mayor cobertura vegetal. Finalmente, para el N_SI, en localidades con ruido alto los valores reportados fueron mayores.

Tabla 1. Modelos lineales generalizados seleccionados para cada variable bajo criterio de información de Akaike

\begin{tabular}{|c|c|c|c|c|}
\hline & Estimador & EE & Valor $\mathrm{t}$ & Valor $p$ \\
\hline F_rango & \multicolumn{4}{|c|}{ Temporada climática + Ruido } \\
\hline Intercepto & 8,024 & $1,24 \mathrm{e}-01$ & 64,499 & $<2 \mathrm{e}-16$ \\
\hline T. seca & $-6,02 e-02$ & $3,00 e-02$ & $-2,006$ & 0,049 \\
\hline Ruido & $3,92 e-03$ & $1,88 e-03$ & 2,084 & 0,041 \\
\hline Fmin_2 & \multicolumn{4}{|c|}{ Cobertura vegetal } \\
\hline Intercepto & 8,347 & $5,71 e-02$ & 146,9 & $<2 \mathrm{e}-16$ \\
\hline Cobertura & $-4,22 e-04$ & $1,76 e-04$ & $-2,4$ & 0,019 \\
\hline F_central & \multicolumn{4}{|c|}{ Temporada climática } \\
\hline Intercepto & 8,318 & $1,28 \mathrm{e}-02$ & 650,195 & $<2 \mathrm{e}-16$ \\
\hline T. seca & $-4,69 e-02$ & $1,82 \mathrm{e}-02$ & $-2,573$ & 0,012 \\
\hline Fmax_SI2 & \multicolumn{4}{|c|}{ Cobertura vegetal + Ruido } \\
\hline Intercepto & 8,312 & $5,61 e-02$ & 148,257 & $<2 \mathrm{e}-16$ \\
\hline Cobertura & $-1,21 e-04$ & $7,68 \mathrm{e}-05$ & $-1,569$ & 0,122 \\
\hline Ruido & 2,13e-03 & $9,09 e-04$ & 2,346 & 0,022 \\
\hline N_SI & \multicolumn{4}{|c|}{ Ruido } \\
\hline Intercepto & $2,36 \mathrm{e}-01$ & $4,42 e-01$ & 0,535 & 0,593 \\
\hline Ruido & 2,31e-02 & $6,74 \mathrm{e}-03$ & 3,425 & 0,001 \\
\hline
\end{tabular}




\section{DISCUSIÓN}

El ruido, la temporada climática y la cobertura vegetal influyeron de forma significativa en algunas variables del canto de Myiothlypis fulvicauda. Como se esperaba, en localidades con ruido alto la frecuencia máxima fue mayor, mientras que en las localidades con mayor cobertura vegetal el canto tuvo menor número de notas. Además, en temporada lluviosa la frecuencia central y el rango de frecuencias fueron mayores.

Esta especie de ave está asociada a cuerpos de agua que generan ruido de considerable intensidad, al que debe adaptar su canto para ser escuchada. Una estrategia ampliamente documentada es el cambio de frecuencias a las que se emite el canto, principalmente un incremento, debido a que el ruido suele concentrarse a bajas frecuencias (Brumm \& Naguib, 2009). En este estudio, la frecuencia máxima fue diferente entre tratamientos de ruido, siendo mayor en localidades de ruido alto; lo mismo ocurre con el rango de frecuencias. Lo anterior podría contribuir a que el canto (o parte de este) se transmita a pesar del ruido ambiental, especialmente en temporada lluviosa, cuando el ruido es mayor (Kirschel et al., 2009). Resultados similares han sido reportados para las especies Pitangus sulphuratus y Turdus leucomelas (Mendes et al., 2017; Mendoza \& ArcePlata, 2012).

Respecto a la frecuencia mínima, esta variable registró valores mayores en las localidades de ruido alto, aunque las diferencias no fueron significativas, posiblemente porque M. fulvicauda canta a altas frecuencias y el porcentaje de solapamiento con el ruido es mínimo (Escalante, 2013); como ocurre con Troglodytes pacificus y el ruido del oleaje o con Cinclus cinclus y el ruido de caídas de agua (Farina \& Gage, 2017; Gough et al., 2014). Otros estudios han reportado que en algunas especies la frecuencia mínima no varía en lugares con ruido, como sucede con Fringilla coelebs cerca de quebradas y cascadas o T. pacificus cerca de oleaje (Brumm \& Slater, 2006; Gough et al., 2014).

Por otro lado, los cantos redundantes tienen mayor probabilidad de ser escuchados en ambientes con altos niveles de ruido (Farina \& Gage, 2017). El canto de M. fulvicauda estuvo formado por hasta 37 notas similares (los ER), que podrían hacerlo redundante. Además, en las localidades con ruido alto se encontró un mayor número de $\mathrm{SI}$, evidenciando así una posible adaptación de esta especie a las condiciones de ruido alto. En individuos de $M$. fulvicauda grabados en Costa Rica se encontraron números de notas similares, hasta $33 \mathrm{ER}$ y $14 \mathrm{SI}$, en condiciones de ruido entre 49 y $60 \mathrm{~dB}$ (Escalante, 2013).

El rango de frecuencias varió entre temporadas climáticas, siendo menor en temporada seca; quizá porque la absorción del sonido es mayor por encima de los 30 으 (Snell-Rood, 2012). Esta situación estaría ocasionando una disminución en dicha variable para que el canto de $M$. fulvicauda sufra menor distorsión tras el aumento de la temperatura (31,6 ㅇ Iluviosa, 32,3 으 seca) (IDEAM, 2019). Según Schäfer et al. (2017), la variación del canto de Cyanistes caeruleus, Parus major y Turdus merula está asociada a variables como la humedad atmosférica, la presión del aire y la temperatura del suelo en áreas urbanas, las cuales estarían relacionadas con la temporada climática. Posiblemente, estas variables afectan el canto de las aves en ambientes naturales. Así mismo, la temporada climática también se asocia con variaciones en la abundancia de insectos como las chicharras, cuya estridulación afecta el canto de algunas aves (Hart et al., 2015), y con la pérdida de hojas en el Bs-T en temporada seca, lo que puede aumentar la probabilidad de detección del canto y la forma en que estos son emitidos o percibidos (Pacifici et al., 2008), variables importantes a considerar en futuros estudios.

Las propiedades del hábitat influyen en la propagación y degradación del sonido. En este estudio, al parecer, la cobertura vegetal favorece cantos con menor número de elementos repetitivos (trino más corto), menor número de notas totales y frecuencias máximas menores en aquellas áreas donde la cobertura vegetal es más densa. Es usual que los cantos en lugares con mayor cobertura vegetal tengan menos trinos que los de áreas abiertas, debido a que estos acumulan mayor reverberación, dificultando así la distinción entre notas (Naguib, 2003). Resultados similares fueron encontrados para Ptilonorhynchus violaceus, puesto que su canto en hábitats con vegetación densa no presentó trinos, o estos fueron cortos (Nicholls \& Goldizen, 2006). Por otra parte, la disminución de la frecuencia máxima de los ER en localidades con mayor cobertura vegetal puede deberse a que en estas áreas la atenuación de las frecuencias altas es mayor (Handford \& Lougheed, 1991). 


\section{CONCLUSIONES}

Algunas variables del canto de Myiothlypis fulvicauda presentaron cambios entre temporadas climáticas y tratamientos de ruido. Principalmente, se observó un aumento en la frecuencia máxima, el rango de frecuencias y el número de silbidos iniciales en localidades con ruido alto ( $>65 \mathrm{~dB}$ ), así como un incremento en el rango de frecuencias y la frecuencia central durante la temporada lluviosa, lo que podría facilitar que el canto de esta ave sea escuchado. En cuanto a la cobertura vegetal, en localidades con mayor densidad se podría estar generando una mayor reverberación y atenuación en el canto, lo que estaría asociado con un menor número de elementos repetitivos y frecuencias máximas más bajas.

\section{REFERENCIAS}

Brumm, H. (2004). The impact of environmental noise on song amplitude in a territorial bird. Journal of Animal Ecology, 73(3), 434-440. https://doi. org/10.1111/j.0021-8790.2004.00814.x

Brumm, H. (2013). Animal communication and noise (vol. 2). Springer Berlin Heidelbert. https://doi. org/10.1007/978-3-642-41494-7

Brumm, H., \& Naguib, M. (2009). Chapter 1. Environmental acoustics and the evolution of bird song. En Advances in the Study of Behavior (1 $1^{\mathrm{a}}$ ed., vol. 40, núm. 09) (pp 1-33). Elsevier Inc. https://doi.org/10.1016/S0065-3454(09)40001-9

Brumm, H., \& Slabbekoorn, H. (2005). Acoustic communication in noise. Advances in the Study of Behavior, 35(05), 151-209. https://doi. org/10.1016/S0065-3454(05)35004-2

Brumm, H., \& Slater, P. J. B. (2006). Ambient noise, motor fatigue, and serial redundancy in chaffinch song. Behavioral Ecology and Sociobiology, 60(4), 475-481. https://doi.org/10.1007/ s00265-006-188y

Catchpole, C., \& Slater, P. (2008). Bird song. Biological themes and variations ( $2^{\mathrm{a}}$ ed.). Cambridge University Press. https://doi.org/10.1017/ CBO9780511754791
Center for Conservation Bioacoustics [CCB]. (2014). Raven Pro: Interactive sound analysis software (Version 1.5) [Computer software]. The Cornell Lab of Ornithology. http://ravensoundsoftware. com/

Douglas, H. D., \& Conner, W. E. (1999). Is there a sound reception window in coastal environments? Evidence from shorebird communication systems. Nature Wissens Chaften, 86(5), 228-230. https://doi.org/10.1007/ s001140050603

Escalante, I. (2013). Comportamiento de canto, descripción de las vocalizaciones y su posible variación geográfica en Costa Rica en Myiothlypis fulvicauda. Zeledonia, 17(1), 35-53. http://www.avesdecostarica.org/ uploads/7/0/1/0/70104897/17-1-000-dig. pdf\#page $=37$

Farina, A., \& Gage, S. H. (2017). Ecoacoustics: The ecological role of sounds. Wiley. https://doi. org/10.1002/9781119230724

Fernández-Juricic, E., Poston, R., De-Collibus, K., Morgan, T., Bastain, B., Martin, C., Jones, K., \& Treminio, R. (2005). Microhabitat selection and singing behavior patterns of male house finches (Carpodacus mexicanus) in urban parks in a heavily urbanized landscape in the Western U.S. Urban Habitats, 3(1), 49-69.

Gill, F. (2007). Ornithology ( $3^{\mathrm{a}}$ ed). W. H. Freeman and Company.

Gough, D. C., Mennill, D. J., \& Nol, E. (2014). Singing seaside: Pacific wrens (Troglodytes pacificus) change their songs in the presence of natural and anthropogenic noise. The Wilson Journal of Ornithology, 126(2), 269-278. https://doi. org/10.1676/13-088.1

Handford, P., \& Lougheed, S. (1991). Variation in duration and frequency characters in the song of the Rufous-collared sparrow, Zonotrichia capensis, with respect to habitat, trill dialects and body size. The Condor, 93(3), 644-658. https:// doi.org/10.2307/1368196 
Hart, P. J., Hall, R., Ray, W., Beck, A., \& Zook, J. (2015). Cicadas impact bird communication in a noisy tropical rainforest. Behavioral Ecology, 26(3), 839842. https://doi.org/10.1093/beheco/arv018

Hilty, S., \& Brown, W. (2001). Guía de las aves de Colombia (H. Álvarez-López, trad.). American Bird Conservancy-ABC.

Holdridge, L. (1967). Life zone ecology. Tropical Science Center.

Instituto de Hidrología, Meteorología y Estudios Ambientales [IDEAM]. (2019, julio 7). Banco de datos estaciones automáticas. IDEAM.

Kirschel, A. N. G., Blumstein, D. T., Cohen, R. E., Buermann, W., Smith, T. B., \& Slabbekoorn, H. (2009). Birdsong tuned to the environment: Green hylia song varies with elevation, tree cover, and noise. Behavioral Ecology, 20(5), 1089-1095. https:// doi.org/10.1093/beheco/arp101

Londoño, C. A. E., \& Fernández, A. E. G. (2011). Protocolo para medir la emisión de ruido generado por fuentes fijas. Revista Ingenierias Universidad de Medellín, 10(18), 51-60. http:// www.scielo.org.co/pdf/rium/v10n18/v10n18a06. pdf

Luther, D. A., Phillips, J., \& Derryberry, E. P. (2016). Not so sexy in the city: Urban birds adjust songs to noise but compromise vocal performance. Behavioral Ecology, 27(1), 332-340. https://doi. org/10.1093/beheco/arv162

Melo, O., \& Vargas, R. (2002). Evaluación ecológica y silvicultural de ecosistemas boscosos. Universidad del Tolima, CRQ, CARDER, Corpocaldas, Cortolima. http://academia.ut.edu. co/images/archivos/Fac_Forestal/Documentos/ LIBROS/evalucaion\%20de\%20ecosistemas\%20 boscosos\%20\%20Rafael\%20vargas\%20y\%20 Omar\%20mel.pdf

Mendes, S., Colino-Rabanal, V. J., \& Peris, S. J. (2017). Adaptación acústica del canto de Turdus leucomelas (Passeriformes: Turdidae) a diferentes niveles de ruido antrópico, en el área metropolitana de Belém, Pará, Brasil. Revista de Biologia Tropical, 65(2), 633-642. https://doi. org/10.15517/rbt.v65i2.25721
Mendoza, A. M., \& Arce-Plata, M. I. (2012). Aproximación al impacto de la perturbación urbana en las vocalizaciones de Pitangus sulphuratus (Tyrannidae) en Santiago de Cali, Valle del Cauca (Colombia). Revista de Ciencias, 16, 19-29. https://doi.org/10.25100/rc.v16i0.500

Morton, E. S. (1975). Ecological sources of selection on avian sounds. The American Naturalist, 109(965), 17-34. https://doi.org/10.1086/282971

Naguib, M. (2003). Reverberation of rapid and slow trills: Implications for signal adaptations to long-range communication. The Journal of the Acoustical Society of America, 113(3), 1749-1756. https://doi.org/10.1121/1.1539050

Narins, P. M., Feng, A. S., Lin, W., Schnitzler, H. U., Denzinger, A., Suthers, R. A., \& Xu, C. (2004). Old World frog and bird vocalizations contain prominent ultrasonic harmonics. The Journal of the Acoustical Society of America, 115(2), 910913. https://doi.org/10.1121/1.1636851

Nicholls, J. A., \& Goldizen, A. W. (2006). Habitat type and density influence vocal signal design in satin bowerbirds. Journal of Animal Ecology, 75(2), 549-558. https://doi. org/10.1111/j.1365-2656.2006.01075.x

Pacifici, K., Simons, T. R., \& Pollock, K. H. (2008). Effects of vegetation and background noise on the detection process in auditory avian point-count surveys. The Auk, 125(3), 600-607. https://doi. org/10.1525/auk.2008.07078

Pijanowski, B. C., Farina, A., Gage, S. H., Dumyahn, S. L., \& Krause, B. L. (2011). What is soundscape ecology? An introduction and overview of an emerging new science. Landscape Ecology 26, 1213-12332. https://doi.org/10.1007/ s10980-011-9600-8

R Core Team. (2019). R: A language and environment for statistical computing. R Foundation for statistical Computing. https://www.R-project.org/

Sánchez-Guzmán, J. N., \& Losada-Prado, S. (2016). Características de la avifauna en un fragmento de bosque húmedo premontano afectado por ruido vehicular. Mutis, 6(2), 7-18. http://dx.doi. org/10.21789/22561498.1147 
Sánchez-Rodríguez, E., López-Mata, L., García-Moya, E., \& Cuevas-Guzmán, R. (2003). Estructura, composición florística y diversidad de especies leñosas de un bosque mesófilo de montaña en la Sierra de Manantlán, Jalisco. Boletín de la Sociedad Botánica de México, 73, 17-34. https:// www.redalyc.org/articulo.oa?id=57707302

Schäfer, J. E., Janocha, M. M., Klaus, S., \& Tietze, D. T. (2017). How weather instead of urbanity measures affects song trait variability in three European passerine bird species. Ecology and Evolution, 7(13), 4868-4880. https://doi. org/10.1002/ece3.3032

Slabbekoorn, H., \& Peet, M. (2003). Birds sing at a higher pitch in urban noise. Nature, 424, 267-268. https://doi.org/10.1038/424267a

Snell-Rood, E. C. (2012). The effect of climate on acoustic signals: Does atmospheric sound absorption matter for bird song and bat echolocation? The Journal of the Acoustical Society of America, 131(2), 1650-1658. https:// doi.org/10.1121/1.3672695
Secretaría de Planeación y TIC [SPT]. (2014). Estadísticas 2011-2014. Gobernación del Tolima/ Universidad de Ibagué. https://www.tolima.gov. co/descargar. php?idFile $=2474$

Swaddle, J. P., \& Page, L. C. (2007). High levels of environmental noise erode pair preferences in zebra finches: Implications for noise pollution. Animal Behaviour, 74(3), 363-368. https://doi. org/10.1016/j.anbehav.2007.01.004

Vargas-Salinas, F., Dorado-Correa, A., \& Amézquita, A. (2014). Microclimate and stream noise predict geographic divergence in the auditory signal of a threatened poison frog. Biotropica, 46(6), 748755. https://doi.org/10.1111/btp.12169 


\title{
Aproximación al tratamiento de aguas residuales del lavado del café con las microalgas Parachlorella kessreli y Desmodesmus armatus
}

\author{
An Approach for Treating Wet Coffee Processing Wastewater Using \\ Microalgae Parachlorella kessreli and Desmodesmus armatus
}

\author{
Iván Alberto Sandoval Salazarac, Mohamed Toufic Darwich Cedeño ${ }^{\mathrm{b}}$, María Del \\ Rosario Castañeda ${ }^{\text {ad }}$, Wilmer Andrés Torres Munara, Luis Carlos Montenegro \\ Ruiz ${ }^{\text {be }}$
}

\footnotetext{
a Grupo de Investigación Yamboró, Servicio Nacional de Aprendizaje (SENA), Pitalito, Huila, Colombia

${ }^{\text {b }}$ Laboratorio de Cultivo de Algas, Facultad de Ciencias, Universidad Nacional de Colombia, Colombia.

chttps://orcid.org/0000-0002-5679-7798

d https://orcid.org/0000-0002-6171-7756

eIcmontenegror@unal.edu.co| https://orcid.org/0000-0002-6662-1833
}

\section{RESUMEN}

Colombia es uno de los mayores productores de café en el mundo, con un mercado que superó los 13,9 millones de sacos en 2020. Sin embargo, en el proceso de beneficio húmedo del café se emplean grandes cantidades de agua, aproximadamente $4.154 .354 \mathrm{~m}^{3}$, en las fases de despulpado, lavado y transporte del grano, generando aguas residuales contaminadas que tienen poco tratamiento antes de ser vertidas a las fuentes hídricas. En el presente trabajo se buscó aislar especies de microalgas que crezcan en estos ambientes, utilizar estas especies en procesos de depuración de aguas residuales del proceso de lavado del café y buscar una aplicación

Citation: Sandoval-Salazar, I. A., Darwich-Cedeño, M. T., Castañeda, M. R., Torres-Munar, W. A. y Montenegro-Ruiz, L. C. (2021). Aproximación al tratamiento de aguas residuales del lavado del café con las microalgas Parachlorella kessreli y Desmodesmus armatus. Mutis, 11(2), 32-43. https:// doi.org/10.21789/22561498.1755

Recibido: 19 de mayo de 2021. Aceptado: 30 de junio de 2021.

Copyright: $\odot 2021$. Sandoval-Salazar, I. A., Darwich-Cedeño, M. T., Castañeda, M. R., Torres-Munar, W. A. y Montenegro-Ruiz, L. C. (2021). This is an openaccess article, which permits unrestricted use, distributions and reproduction in any medium, provided the original author and source are credited.

Competing Interests: The authors have no conflict of interest. de la biomasa resultante. Para la obtención de las cepas de microalgas se utilizaron técnicas de agotamiento. Con el fin de evaluar su capacidad de depuración, estas fueron cultivadas en agua residual de diferentes concentraciones bajo condiciones de laboratorio, determinando la remoción de materia orgánica. Adicionalmente, se caracterizó bioquímicamente la biomasa algal para determinar posibles usos biotecnológicos o industriales. Se encontró dos Chloroficeaes cocales, Parachlorella kessreli y Desmodesmus armatus, las cuales demostraron una alta eficiencia en la remoción de nitrógeno total (> 50 \%) y fósforo total 